Trends Pharmacol Sci. 2015 June ; 36(6): 374-383. doi:10.1016/j.tips.2015.03.003.

\title{
Hypoxia-Inducible Factors in Cancer Stem Cells and Inflammation
}

\author{
Gong Peng ${ }^{1}$ and Yang Liu',2 \\ ${ }^{1}$ Institute of Translational Medicine, The First Hospital, Jilin University, Changchun, China \\ ${ }^{2}$ Center for Cancer and Immunology Research, Children's Research Institute, Children's National \\ Medical Center, Washington, DC, USA
}

\begin{abstract}
Hypoxia-inducible factors (HIF) mediate metabolic switch in cells in hypoxic environments, including those in both normal and malignant tissues with limited supplies of oxygen.

Paradoxically, recent studies have shown that cancer stem cells and activated immune effector cells exhibit high HIF activity in normoxic environments and that HIF activity is critical in maintenance of cancer stem cells as well as differentiation and function of inflammatory cells. Since inflammation and cancer stem cells are two major barriers to effective cancer therapy, targeting HIF may provide a new approach for the ultimate challenges.
\end{abstract}

\section{Keywords}

Leukemia-initiating cells; tumor microenvironment; myeloid-derived suppressor cells; dendritic cells; tumor-associated macrophages; cancer immunotherapy

\section{Challenges in targeting tumor microenvironment}

An important paradigm shift in cancer research has been the realization that cancer tissue is not a homogenous population of clonally expanded cancer cells [1-3]. It has been established in multiple cancer types that cancer cells are hierarchical: while a small subset of cancer stem cells have a high capacity for self-renewal and are responsible for initiating cancer, the bulk of cancer cells lack self-renewal and cancer-initiating capacity [3, 4]. Perhaps because conventional therapeutic approaches were not developed to target cancer stem cells, many cancer stem cells are enriched by conventional cancer therapy $[5,6]$. The ineffective elimination of cancer stem cells is considered as a major cause for cancer relapses following conventional therapy, and thus presents as a major barrier to effective cancer treatment [7].

\footnotetext{
() 2015 Published by Elsevier Ltd.
}

Publisher's Disclaimer: This is a PDF file of an unedited manuscript that has been accepted for publication. As a service to our customers we are providing this early version of the manuscript. The manuscript will undergo copyediting, typesetting, and review of the resulting proof before it is published in its final citable form. Please note that during the production process errors may be discovered which could affect the content, and all legal disclaimers that apply to the journal pertain. 
In addition to heterogeneity among transformed cancer cells, cancer tissues also consist of non-cancerous host cells [1,2]. Together, cancer cells and host cells form a tumor microenvironment that enables tumor initiation and progression. The dependence of cancer on the host cells suggest that these host cells may also be targeted for cancer therapy. Again, since conventional cancer therapy was developed without emphasizing tumor microenvironment, a major new focus for cancer therapy is to limit cancer development by targeting the tumor microenvironment.

Based on these considerations, it would be of interest to identify druggable targets critical for cancer stem cells and tumor-promoting microenvironments. In this context, we and others have revealed selective activation of the HIF pathway and metabolic switch of cancer stem cells [8-10]. Remarkably, recent studies have demonstrated that HIF may play a major role in inflammation, including the adaptive and innate inflammatory response [11] [12, 13]. The shared requirement for HIF in cancer stem cells and inflammatory cells raised the interesting prospect that cancer stem cells and inflammation, two important challenges in cancer therapy, may be addressed by targeting HIF. Here we review the critical role for HIF in immunology and cancer biology, with focus on potential cross-fertilization of HIF research on cancer cells and host inflammatory cells, and explore the translational potential of this new concept. Readers are referred to outstanding recent reviews for the general concept and involvement of HIF in cancer biology and immunology [11, 14, 15].

\section{HIF and cancer: an overview}

The modes of energy production in a cell are usually dictated by the oxygen levels in the environment: oxidative phosphorylation occurs in a well-oxygenated environment (normoxia), while glycolysis is switched on when oxygen levels drop below 1\% (hypoxia) [15]. In a search for the fundamental mechanism of the oxygen-mediated metabolic switch, Gregg Semenza and colleagues identified HIF-1a, an oxygen-senstive transcriptional activator [16]. Recent studies suggest that by directly regulating expression and activity of pyruvate kinase muscle isozyme 2 (PKM2), HIF-1a can serve as a master switch for oxygen-regulation in cellular metabolism [17].

As illustrated in Figure 1, HIF is a heterodimer consisting of $\alpha$ and $\beta$ subunits. The heterodimers translocate into the nucleus where they interact with specific DNA sequences called HIF-responsive elements (HREs). By binding to the HRE, HIF may either activate or repress gene expression. At least three different genes have been identified that encode a subunit of HIF, called HIF1a, HIF2a and HIF3a. All three HIFa subsunits heterodimerize with a HIF- $1 \beta$ subunit and are subject to posttranslational regulations that are dictated by oxygen concentration in the environment. Although HIF-3a lacks a transactivation domain and is generally considered to be a negative regulator for HIF-1a and HIF-2a function, a notable exception was reported recently [18]. Despite the general similarity in regulation and function between HIF-1a and HIF-2a, they differ in their sensitivity to oxygen-deprivation, target gene binding and tissue distribution [14].

The critical role of HIF in cancer biology was established when a major renal tumor suppressor gene VHL was identified as the E3 ligase responsible ubiqutinylation of HIF-1a

Trends Pharmacol Sci. Author manuscript; available in PMC 2016 June 01. 
and HIF-2a [19-23]. VHL recognizes hydroxylated residues at Pro402 and/or Pro562 in HIF-1a and Pro 405 and Pro 531 in HIF-2a, by means of the prolyl hydroxylase domain protein (PHD) [24]. VHL is inactivated in most renal cancer samples, leading to increased expression of HIF-1a and HIF-2a protein [19-23]. Mutations of PHD protein encoding genes, EGLN1 (PHD2), EGLN2 (PHD1), and EGLN3 (PHD3) have been observed in various cancers at low frequency (http://www.cbioportal.org/). The function of PHD is enhanced by isocitrate dehydrogenase 1 (IDH1) and/or IDH2 [25, 26], which are mutated at a combined frequency of $\sim 15 \%$ in patients with acute myeloid leukemia (AML) [27-30] and low grade glioma or glioblastoma [31]. Two reports suggested that IDH mutations lead to increase in HIF-1a accumulation [25, 26], while a more recent study suggests otherwise [32].

Over-expression of either HIF-1a and/or HIF-2a is a marker for poor prognosis in the majority of the cancer types tested, including common cancers, such as breast, prostate, colon, hepatocellular, pancreatic, brain, and ovarian cancers, as well as a host of less common cancers [14]. In transgenic mouse cancer models, heterozygous deletion of Hifla reduced the growth of thymic lymphoma [33], while shRNA silencing of Hifla and overexpression of VHL strongly reduced leukemia-initiating activity [9].

Despite the overwhelming association between HIF levels in cancer tissue and poor prognosis of cancer patients, conflicting reports have emerged in renal cancer, where overexpression of HIF-1a has been associated with either poor or favorable prognosis, depending on the methods used to evaluate HIF-1a levels [34, 35]. In the mouse cancer models, recent studies suggest that while local deletion of Hifla in lung tissue had no impact on Kras-driven lung cancer, deletion of Hif2a paradoxically accelerated lung cancer development [36]. More recently, broad deletion of Hifla in adult mice, including that in the cells that give rise to leukemia, promote, rather than suppress Mll-AF9a-induced leukemia [37]. These contradicting observations may be explained by the broad spectrum of HIF target genes: the cancer-promoting effect is exemplified by HIF-mediated induction and function of PKM2, vascular endothelial cell growth factor (VEGF) and multidrug resistance gene (MDR1), while that of its function as a tumor suppressor can be explained by both the transcriptional and non-transcriptional activity of HIF.

\section{PKM2 and metabolic switch in cancer cells}

PKM2 is the final enzyme in glycolysis and consists of two isoforms that arise from alternative splicing. Most tissues express the PKM1 isoform with products directed into oxidative phosophorylation to efficiently produce ATP. PKM2, by contrast, may exist either in tetrameric or dimeric forms to direct oxidative phosphorylation or glycolysis, respectively. Because PKM2 exists predominantly in its dimeric form in cancer cells, it contributes to a high rate of aerobic glycolysis in cancer cells regardless of hypoxia, a phenomenon known as the Warburg effect. HIF regulates PKM2 through two mechanisms [17]: first, HIF-1a can stimulate expression of PKM2. Second, PKM2 and HIF-1a form heterodimers and migrate into the nuclei, where they act as a master switch to over-express genes crucial for a robust glycolysis that produces both energy and metabolic intermediates for biosynthesis. Therefore, HIF-1a plays a critical role for metabolic switch in cancer cells.

Trends Pharmacol Sci. Author manuscript; available in PMC 2016 June 01. 
In addition to PKM2, HIF-1a has been shown to antagonize cMyc activity by inducing expression of MX11, thus reducing mitochondrial biogenesis [38].

\section{VEGF and cancer neoangiogenesis}

The increase in tumor volume demands a corresponding increase in angiogenesis. By regulating VEGF expression, both cellular and viral oncogenes not only regulate the growth of cancer cells, but also allow cancer progression in the host. Optimal VEGF expression was found to depend on both hypoxia and oncogenes [39]. These observations led to the identification of HIF-1a as a major regulator of VEGF expression [40, 41]. HIF-1ap300/CBP complex binds to a HIF-responsive element in the 5' promoter region of the gene encoding $(V E G F)[40,41]$. This HRE sequence was specifically targeted by echinomycin [9, 42].

MDR1

The link between HIF and MDR1 was first reported in non-transformed endothelial and epithelial cell lines [43]. The authors showed that HIFla is responsible for hypoxia-induced $M D R 1$ expression through stimulation of the MDRI promoter sequence. Subsequently, it was shown in head and neck cancer cell lines that inhibition of HIF-1a increased cancer cell sensitivity to paclitaxel [44]. The contribution of HIF to multidrug resistance is now substantiated in many types of cancer, including gastric cancer, colon cancer, multiple myeloma, AML, laryngeal cancer, and sacral chordoma [45-51]. These observations explained the poor prognosis of patients with cancer over-expressing HIF and suggest an important role for HIF inhibitors in combinatorial cancer therapy.

\section{Tumor growth inhibition by HIF-1a}

Despite compelling evidence for oncogenic effect of the HIF family members, over activation of HIF may cause growth arrest and/or apoptosis. At least three mechanisms have been proposed to explain tumor suppressor activity of HIF-1a. First, HIF-1a has been shown to stabilize p53, perhaps by binding to MDM2 [52]. Second, among HIF-1a targets are genes capable of inducing apoptosis, including RPT801 [53], NIP3 [54, 55] and NIX [55]. Third, HIF-1a has been shown to antagonize $M y c$ by preventing its repression of $\mathrm{p} 21$ [56]. In combination, these mechanisms may reconcile the inconsistencies in the mouse genetic data which suggest that the Hif1a gene may either promote or suppress tumor growth depending on the tumor types investigated [33] [37].

\section{HIF and cancer stem cells}

An important advance in cancer biology was made with the demonstration that somatically transformed cancer cells are heterogeneous in establishing cancer in a new host. In many solid and hematological cancers, a small subset of cancer-initiating cells can be prospectively isolated based on either cell-surface phenotypes of their ability to exclude fluorescent dyes (side population). Although it has been suggested that therapeutic elimination of cancer stem cells may hold the key to reduce cancer relapse and drug resistance, few druggable targets have been identified that provide a means for selective 
elimination of cancer stem cells [57-59] [60]. As reviewed below, accumulating data suggest that HIF may emerge as a long-sought target.

\section{Glioma stem cells and HIF}

The involvement of HIF pathway in glioma stem cells was first reported by Li et al. [8]. Using xenograft glioma initiating and in vitro tumorosphere formation assays and CD133 as the cancer stem cell markers, the authors observed significant enhancement of stem cell activity when the stem cells were cultured under a hypoxic environment. Interestingly, the stem cell activity under both normoxic and hypoxic environments are reduced when either HIF $1 a$ or HIF $2 a$ are silenced by shRNA. Since HIF $2 a$ mRNA levels correlate with glioma activity and glioma progression and prognosis, the authors emphasized that HIF2a is critical for the glioma stem cell activity. However, it should be noted that small hairpin RNA (shRNA) silencing showed an equally important role for HIFla in cancer stem cell activity. The lack of correlation between HIFIa mRNA levels and stem cell activity may simply reflect the fact that HIF-1a protein levels are regulated by post-transcriptional mechanisms. The HIF targets responsible for CSC activity remain to be identified.

\section{Acute lymphocytic leukemia (ALL)}

Early work on cancer stem cells was criticized for using xenograft transplantation as the CSC activity (tumor-initiating activity) assayed in the xenogeneic host may be artificially affected by rejection of human cells by the innate immunity of the recipient mice [61]. Wang and colleagues used a syngeneic transplantation model to demonstrate that the c$\mathrm{Kit}^{+} \mathrm{Sca}-1^{+}$population in their mouse model of ALL are the leukemia initiating cells [9]. Since the c-Kit ${ }^{+} \mathrm{Sca}-1^{+}$cells are the necessary and sufficient population for an in vitro colony-forming unit (CFU), they used this model to identify inhibitors that may target the ALL stem cells. They found that echinomycin, a natural product that binds to HRE and thus inhibits HIF activity efficiently eliminated the CFU with an IC50 of 30-100 pM. In vivo, low dose administration of echinomycin $\left(10 \mu \mathrm{g} / \mathrm{kg}\right.$ or $\left.30 \mu \mathrm{g} / \mathrm{m}^{2}\right)$ cured $100 \%$ of syngeneic mice that received lethal doses of leukemia cells. The involvement of HIF-1a is substantiated by three lines of evidence. First, Hifla, but not Hif2a gene, is expressed at substantially higher levels in the leukemia stem cell population than the bulk of leukemia blasts. Second, shRNA silencing of Hifla reduced CFU and leukemia-initiating activity. Third, Hif-1a was found to enhance Notch signaling by preventing negative feedback regulation of the Hesl gene, a key Notch target known to be involved in stem cell selfrenewal. However, it remains to be established whether up-regulation of Hes 1 is responsible for HIF-mediated maintenance of LSC.

Using a HIF reporter, Wang et al. showed that under normoxic conditions, HIF is active exclusively within the c-Kit ${ }^{+} \mathrm{Sca}-1^{+}$leukemia stem cell (LSC) subset [9]. This high HIF activity was due to selective loss of VHL-expression in the stem cell subset, which is critical for LSC function as ectopic expression of Vhl eliminates the LSC [9]. 
Historically, prospective identification of AML stem cells by John Dick and colleagues in 1994 marked the revival of the cancer stem cell concept [4]. Several groups have shown that the abundance of AML stem cells is a biomarker for poor prognosis [62-64]. Gene expression signatures derived from comparison of AML stem cells and AML blasts have proven valuable in predicting the outcome of newly diagnosed patients [65]. An important prediction of the cancer stem cell concept is that therapeutics that selectively eliminate stem cells should prevent drug resistance and cancer relapse, the two most pressing issues in cancer therapy. Since echinomycin can selectively eliminate AML stem cells [9], the planned clinical trials using echinomycin [66] may provide an opportunity to test this concept

Using the AML stem cell markers identified by Dick's group, Wang and colleagues [9] showed that HIFla mRNA and protein is over-expressed in the human AML stem cells in comparison to the bulk of AML blasts. In both naïve and treated AML samples, echinomycin efficiently inhibits CFU activity, with an IC50 of about $100 \mathrm{pM}$. More importantly, echinomycin is 100-1000 fold more efficient in inducing apoptosis of the $\mathrm{CD} 34^{+} \mathrm{CD} 8^{-}$AML stem cells in comparison to the bulk of AML blasts. To test the therapeutic potential, the authors established human AML in the NOD.SCID mice according to the methods described by the Dick laboratory [4] and treated them with a low dose of echinomycin [9]. Short-term treatment with echinomycin not only reduced leukemia blast burden, but also preferentially reduced the frequency of AML stem cells within the leukemia cells, marking a major difference with the conventional therapeutics. As evidence of functional inactivating AML stem cells, the remaining AML blast(s) could no longer initiate AML in the new host.

To test whether echinomycin can be used to treat relapsed AML, Wang and coworkers[67] used the relapsed AML from mice with heterozygous knock-ins of two genes that are frequently mutated in human AML: FLT3 $3^{I T D}$ and $M L L^{P T D}\left(M l l^{\mathrm{PTD} / \mathrm{WT}}: F l t 3^{\mathrm{ITD} / \mathrm{WT}}\right)$. The $M l l^{\mathrm{PTD} / \mathrm{WT}}: F l t 3^{\mathrm{ITD} / \mathrm{WT}}$ mice developed spontaneous AML and responded to treatment with a DNA hypomethylating agent and/or a histone deacetylase (HDAC) inhibitor; however, the AML invariably relapsed [68]. The authors[67] transplanted the relapsed AML from CD45.2 mice into CD45.1 mice and followed the expansion and therapeutic response using CD45.2 as a leukemia marker [64]. They observed that short-term treatment with echinomycin treatment cured $40-60 \%$ of mice with a high burden of relapsed AML at the time of treatment. Again the bone marrow from the cured mice did not harbor dormant AML stem cells as was evidenced by its inability to initiate AML in the new hosts. Surprisingly, echinomycin-treated bone marrow cells are fully competent in hematopoiesis in transplantation. Therefore, therapeutic elimination of cancer stem cells can be achieved in relapsed AML without significant adverse effect on tissue stem cells. Thus, although cancer stem cells and tissue stem cells may share self-renewal programs, therapeutic elimination of cancer stem cells can be achieved without harming tissue homeostasis. 


\section{Chronic myeloid leukemia}

Chronic myeloid leukemia (CML) is a clonal myeloproliferative disorder that results from an acquired genetic change in a single hemopoietic stem cell. The hallmark of CML is generation of BCR-ABL chimera protein, a constitutively active tyrosine kinase, as a result of gene translocation [69]. Induction of BCR-ABL induces expression of HIF-1a and its target gene VEGF [70]. Kinase inhibitors (such as imatinib) failed to eradicate CML LSCs. Instead, the targeted therapy selected for imatinib-resistant cells with high levels of BCRABL and active HIF-1a, resulting in anaerobic glycolysis and increased survival in vitro [71]. Using a mouse model of CML, Zhang et al. showed that Hifla-deficient HSPCs expressing BCR-ABL failed to generate CML in secondary recipients. Deletion of Hifla impairs the propagation of CML by impairing cell-cycle progression and inducing apoptosis of LSCs [72]. In addition, recent studies also showed that HIF1-a supports maintenance of CML LSCs despite effective BCR-ABL1 inhibition in hypoxic environments [73]. These observations indicated that Hif-1a is crucial for maintenance of CML LSCs.

Taken together, the above examples illustrate that HIF plays a critical role in the stem cells for both solid tumors, and hematological malignancies, serving as a druggable target for therapeutic elimination of cancer stem cells. The fact that HIF is active in cancer stem cells regardless of hypoxia suggests that these cells have acquired mechanisms to protect HIF under normoxic conditions.

\section{Breast cancer stem cells (BCSCs)}

Breast cancer is the first solid tumor in which cancer stem cells were prospectively isolated [3]. Using xenograft model of human breast cancer cell lines, Conley et al. observed that anti-angiogenic agents increased the proportion of BCSCs by inducing hypoxia. The impact of hypoxia is mediated by HIF-1a, but not HIF- 2 a, and correlates with Wnt signaling in BCSC [74]. Using the mouse mammary tumor virus polyoma virus middle T (MMTVPyMT) oncogene-induced breast cancer model, Schwab et al. [75] demonstrated that conditional deletion of the Hifla gene in the mammary epithelial cells cause reduced tumor growth and metastasis. Interestingly, this depressed tumor growth and metastasis corresponds to a reduction in the number and function of BCSC [75].

A key issue is how HIF-1a induces BCSC. One key regulator of BCSC activity is a Hippo pathway effector molecule called TAZ [76]. By comparing gene expression profile among 1600 cases of human breast cancer samples, Xiang et al [77] showed a significant correlation between known HIF target genes and TAZ, raising the intriguing possibility that TAZ may be a direct target of HIF-1a. This hypothesis is validated by shRNA silencing, chromatin-immunoprecipitation, promoter activity, TAZ target gene co-expression and hypoxia responses. In addition to controlling TAZ expression, HIF-1a also activates transcription of the SIAHI gene, which encodes a ubiquitin ligase that is required for proteasome-dependent degradation of LATS2. Degradation of LATS2 allowed nuclear localization of TAZ. Apart from expression and function of TAZ, hypoxia also regulates the interaction between BCSC and mesenchymal stem cells (MSC) and tumor-associated macrophages (TAM) through two feed-forward mechanisms [78]. First, HIF-1a induces expression of CXCL16 to recruit MSC. Second, the MSC produces CCL5 to recruit TAM. 
Therefore, BCSC-intrinsic signaling not only maintains the number and function of the $\mathrm{BCSC}$, but also drives formation of the tumor microenvironment.

\section{HIF in adaptive and innate inflammation}

Hypoxia-resistant HIF activity is not limited to cancer cells. Accumulating evidence shows that HIFs play a role in regulation of innate and adaptive immune cells that normally reside in normoxic environments. Activated T cells express two isoforms of HIF-1a: short form called 1.1 and a longer form called 1.2 [79]. The shorter 1.1 form appears to suppress production of inflammatory cytokines by $\mathrm{T}$ cells [80]. Accumulating data demonstrates that HIF is a key regulator for Treg/TH17 development, differentiation and function of cytotoxic T lymphocytes, (CTL), and innate immune effectors such as dendritic cells, neutrophils and myeloid-derived suppressor cells (MDSCs).

\section{T helper 17 (TH17) /Treg cell differentiation}

Although Th17 and Treg cells share important pathways in their differentiation from naïve $\mathrm{T}$ cells, they eventually bifurcate into distinct phenotypes with opposite activities, with TH17 cells being pro-inflammatory and Tregs being anti-inflammatory. Recent studies suggest that the functional switch between these cell types may be mediated by HIF-1a.

HIF-1a activates $R O R \gamma t$ transcription and forms a tertiary complex with ROR $\gamma \mathrm{t}$ and $\mathrm{p} 300$ to activate the IL-17A gene. By contrast, Hif-1a binds to Foxp3 and causes its degradation, resulting in a blockade of Treg differentiation. As a result of altered Th17/Treg differentiation, mice with HIF-1a-deficient $\mathrm{T}$ are resistant to experimental autoimmune encephalomyelitis [81]. When naïve T cells were cultured in the presence of TGF- $\beta$ and IL6, they showed upregulation of the glycolytic activity and induction of glycolytic enzymes. A critical role for glycolysis in Th17 development is suggested as blocking glycolysis with 2-dexoglucose inhibits TH17 cell development. Consistent with a critical role for HIF in Th17 differentiation, HIF-1a was selectively expressed in TH17 cells, while Hifla deficiency inhibits TH17 differentiation [82]. Function of HIF-1a in TH17 was not restricted to regulation of the metabolic switch. HIF-1a controlled expression of antiapoptotic Bcl-2 family genes in collaboration with Notch signaling and thus supports TH17 survival in human samples [83]. Taken together, these data highlight HIF-1a-dependent pathways in regulating the balance between differentiation of TH17 and Treg cells.

Despite the in vitro effect of HIF-1a on Treg differentiation, the inhibitory function of HIF on Treg function in the tumor microenvironment remains to be substantiated. Surprisingly, HIF promotes differentiation of $\mathrm{CD} 4^{+} / \mathrm{CD} 25^{+}$Treg cells in the case of hypoxia caused by rapid tumor progression [84]. Tregs may be enriched in cancer tissue by HIF-1a-dependent production of chemokines, such as CCL-28 [85]. Additional studies are needed to determine if the apparent contradiction was attributable to tumor microenvironment, and if so, how tumor microenvironment alters the role for HIF-1a in Treg differentiation.

\section{Cytotoxic T lymphocyte differentiation and function}

CTLs are the key adaptive effectors in elimination of intracellular pathogens and tumor cells. HIF-1a determines CTL fate by regulating transcription of genes encoding glucose 
transporters, rate-limiting glycolytic enzymes, cytolytic effector molecules, and essential chemokine and adhesion receptors that regulate $\mathrm{T}$ cell trafficking [86]. Activated $\mathrm{T}$ cells express high levels of HIF-1a proteins even under normoxia, although hypoxia further elevates the HIF-1a [87] [86]. The induction of HIF-1a is controlled by mTORC1 [86]. Genetic studies in mice demonstrated that deletion of $V h l$ in CTLs inhibits persistent viral infection and neoplastic growth, while that of Hifla attenuates CTL effector function during viral infection [87]. Surprisingly, deletion of Hifla in activated T cells also enhanced production of inflammatory cytokines. These data argue for a negative regulatory role for HIF-1a in T cell-mediated inflammatory responses [80, 88]. Further studies are needed to shed light on T cell-intrinsic HIF-1a function in the tumor microenvironment.

\section{Dendritic cells (DCs) activation}

As the primary antigen-presenting cells, DCs play crucial role in linking the innate and the adaptive immune systems. LPS and hypoxia lead to increased glycolytic activity and increased DC maturation. The role for HIF-1a is confirmed as knocking down of HIF-1a in DCs significantly reduced glucose utility and inhibited DC maturation as evidenced by reduced stimulation of allogeneic T cells [89]. While the role for HIF-1a in tumorinfiltrating DC has not been investigated, the induction of B7H1/PD-L1 by HIF-1a [90, 91] and the immune suppression by B7H1-expressing myeloid DC in human cancer [92] suggest that HIF-1a expressed in the tumor-infiltrating DC may contribute to the immunesuppressive tumor microenvironment.

\section{Tumor-associated myeloid suppressor cells}

Tumor growth alters myeloid development in the host, with significant phenotypic and functional changes in the myeloid compartment [93]. A major change in the tumor-bearing host and the tumor microenvironment is the expansion of myeloid suppressor cells, including tumor-associated macrophages (TAM), myeloid-derived suppressor cells (MDSC) and myeloid dendritic cells (mDC) [93]. Accumulating data demonstrate that HIF-1a contributes to expansion, differentiation and effector function of the myeloid suppressor cells within the tumor microenvironment.

As discussed above, HIF-1a may contribute to mDC-mediated immune suppression by regulating expression of B7H1/PD-L1. Both TAM and MDSC suppress tumor immunity by producing soluble factors such as nitric oxide (NO) and reactive oxygen species (ROS) [94] [93], and through expression of cell-surface proteins such as B7H1/PD-L1. HIF-1a, but not HIF-2a, upregulates the expression of B7H1/PD-L1 by binding to the HRE of the PD-L1 proximal promoter to enhance expression of PD-L1 [90]. In addition, HIF-1a is necessary for optimal expression of arginase and iNOs in both TAM and MDSC and is thus responsible for NO production [95]. Interestingly, MDSC have been shown to differentiate into TAM in the tumor microenvironment, and this process is requires expression of HIF-1a MDSC [95].

The contribution of HIF to tumor microenvironment has been demonstrated mostly using transplantable tumor models. As a notable exception, myeloid-specific HIF-1a-deletion a transgenic model of breast cancer reduces tumor growth and progression [96]. This is 
achieved without alternation of VEGF-A levels and vascularization. To test whether this deletion affects macrophage functions, the authors compared WT and Hifla $a^{-/}$bone marrow-derived macrophages under normoxic and hypoxic culture conditions. The authors observed that hypoxia exacerbated inhibition of $\mathrm{T}$ cell proliferation and that such exacerbation is Hifla-dependent. Since TAM are known to inhibit T cell function [93], it would be of interest to determine whether the delay in tumor growth was attributable to Hifla deletion in TAM.

\section{Epigenetic and genetic mechanisms for HIF activities under normoxia: cross-fertilization between cancer biology and immunology}

The critical role for HIF in cancer cell metabolism, cancer stem cell function, and immunity raised a fundamental but largely unanswered question: how is HIF activity maintained in circulating leukemia stem cells, cancer cells and hematopoietic cells in a normoxic environment? Accumulating data indicate that HIF activation is stimulated by increased transcription and translation of the HIFla gene and inactivation of oxygen-mediated HIF degradation, through both genetic and epigenetic mechanisms.

\section{Genetic mechanisms taught by cancer: the AML example}

The accumulation of HIF1a protein and its transcriptional activity are strictly regulated. As shown in Figure 2, many of these mechanisms are targeted in AML, and a number of genomic alterations in AML have either been shown or have the potential to enhance HIF activity. First, AML-associated mutations seem to target the canonical HIF degradation pathway. Under normoxia, HIF-1a is degraded by VHL, which recognizes HIF-1a when it is hydroxylated at Pro402 and/or Pro562 by the prolyl hydroxylase domain protein (PHD) [24]. Mutations of gene encoding PHD have observed in AML only at low frequency. However, the function of PHD is enhanced IDH1 and/or IDH2 [25, 26], which are mutated at a combined frequency of $\sim 15 \%$ in AML patients [27-30]. One study showed that IDH mutations increase HIF1a accumulation [25, 26]; while a later study suggested an opposite effect [32]. The differences between the two remain to be reconciled. Second, FLT3-ITD (internal tandem repeats) and TKD (tyrosine kinase domain) mutations activate FLT3 [97, 98]. Since FLT3 signaling activates the PI3K-mTOR pathway [99], and since mTOR activation increases HIF1a protein accumulation [100-103], it would be intriguing to test whether FLT3 mutations cause HIF activation in AML. Third, loss of TP53 function in tumor cells has been shown to cause defective MDM2-mediated degradation of HIF-1a [104]. Since p53 mutations are observed in approximately 10\% of AML samples, and since this mutation is associated with devastating prognosis [105], it would be of great interest to determine whether this mutation contributes to increased HIF-1a accumulation in AML. Given the fact that MDM2 is an E3 ligase for HIF-1a [106, 107], p53 mutations may increase HIF-1a levels by decreasing MDM2. Fourth, NPM1 is mutated in 30-40\% of AML samples that have a normal karyotype [108, 109]. Since NPM1 sequesters and protects p14ARF against degradation [110], NPM mutations may stimulate HIF activities by inactivating the p14ARF-mediated aberrant delocalization of HIF1a [111].

Trends Pharmacol Sci. Author manuscript; available in PMC 2016 June 01. 


\section{Epigenetic mechanism of HIF1a activation: unanswered questions from cancer stem cells and immune effector cells}

As the offspring of cancer stem cells, cancer cells should have inherited genetic alterations that enable oxygen-resistant HIF activity. Therefore, for cancers that show selective activation of HIF within the cancer stem cell compartment, including AML, ALL, CLL and glioma, genetic alterations in HIF regulators do not offer full explanation. There are at least two mechanisms for selective HIF-1a activation in mouse leukemia stem cells [9]: increased Hifla mRNA accumulation and lack of expression of Vhl. Both Hifla upregulation and Vhl downregulation are necessary for the maintenance of LSC. Since LSCs are a self-renewing population that also "differentiates" into cancer cells, it is of great interest to determine the epigenetic mechanism that ensures the heritable gene expression pattern of Hifla and Vhl and how this pattern is lost when the LSCs "differentiate" into cancer cells. Elucidation of this mechanism will also help to formally demonstrate the existence of epigenetic programs that maintain the LSC activity by selective activation of HIF-1a in this compartment.

Similarly, how oxygen-resistant HIF-1a activity is induced in the immune effector cells remains largely unexplained. Accumulating data suggest that activation of either T-cell receptor [81, 82, 112] or Toll-like receptors (TLR) [113] induce accumulation of Hifla mRNA, perhaps through suppression of miR-200 [112]. However, additional mechanisms are needed to explain oxygen-resistant HIF-1a activity. AKT-PI3K pathway induces accumulation of HIF-1a through activation of mTOR to increase translation of HIF-1a [114]. In addition, mTOR activation prevents oxygen-induced HIF-1a degradation [102]. In the latter case, the mTOR-induced HIF protection against degradation requires functional interaction between mTOR and the HIF-1a domain that is also involved in hydroxylation of HIF-1a. Therefore, mTOR may inhibit HIF degradation through a yet undefined mechanism.

Recent studies demonstrated that, much like adaptive immunity, innate effector cells may be also capable of immune memory, i.e., they can be trained to mount a more robust or much reduced recall response $[12,13]$. For instance, stimulation of monocytes with glucan trained the monocytes to mount a more robust innate immune response to secondary stimulation in vitro and increased host resistance to infections by Candida albicans and S. aureus [12]. This training is associated with extensive epigenetic alterations in the monocytes [13]. Surprisingly, the training is associated with a metabolic switch from oxidative phosphorylation to glycolysis [12]. Given the critical role for HIF-1a in the metabolic switch, researchers used a genetic model to determine the involvement of HIF-1a in the recall response of innate effectors [12]. Indeed, targeted mutation of Hifla in myeloid cells is sufficient to ablate trained immunity. The critical role for HIF-1a in trained immunity and the extensive epigenetic reprogramming during the process raised an interesting issue as to whether epigenetic mechanisms may be directly responsible for oxygen-resistant HIF activation in monocytes.

Taken together, in both immune effectors and cancer stem cells, a steady state of oxygenresistant HIF-1a accumulation has been achieved. A major challenge remains in defining how this crucial state is achieved through non-genetic, perhaps epigenetic,mechanisms.

Trends Pharmacol Sci. Author manuscript; available in PMC 2016 June 01. 


\section{Concluding remarks}

HIF was discovered in the process of investigating how living organisms adjust to varying oxygen environment [15]. More recent studies have demonstrated that this pathway is operative even under normoxic environment and that such oxygen-resistant function is critical in cancer biology and immunology. HIF activation is responsible for a metabolic switch that allows more sustained proliferation with less DNA damage. These features are important for the maintenance of cancer stem cells. In the immune system, activation and/or function re-programming of immune effectors is often imprinted through oxygen-resistant HIF accumulation. The converging feature suggests cross-fertilization between cancer biologists and immunologists may bring new insights on the mechanism of HIF regulation and new approaches for cancer therapy.

As summarized in Figure 3, cancer cells and immune effectors are the major components that constitute the tumor microenvironment. In this environment, the immune system not only fails to defend the host against cancer but often actively aid carcinogenesis, with functions ranging from promoting cancer stem cell activity [115, 116], angiogenesis [117], to facilitating malignant transformation [118] and metastasis [119]. Therefore, effective control of the tumor microenvironment will likely complement the traditional approach of cancer therapy, aiming at eliminating cancer cells. Since most contributors to the tumorpromoting microenvironment rely on HIF, it is likely that targeting HIF will not only aid in the therapeutic elimination of cancer stem cells, but also result in depriving cancer cells of a habitable microenvironment.

\section{Acknowledgement}

We thank Mr. Christopher Bailey for editorial assistance. This work is supported by Jilin University First Hospital and grants from National Cancer Institute, USA (CA183030 and CA171972 to YL).

\section{References}

1. Hanahan D, Weinberg RA. The hallmarks of cancer. Cell. 2000; 100:57-70. [PubMed: 10647931]

2. Hanahan D, Weinberg RA. Hallmarks of cancer: the next generation. Cell. 2011; 144:646-674. [PubMed: 21376230]

3. Al-Hajj M, et al. Prospective identification of tumorigenic breast cancer cells. Proc. Natl. Acad. Sci. U. S. A. 2003; 100:3983-3988. [PubMed: 12629218]

4. Lapidot $\mathrm{T}$, et al. A cell initiating human acute myeloid leukaemia after transplantation into SCID mice. Nature. 1994; 367:645-648. [PubMed: 7509044]

5. Bao S, et al. Glioma stem cells promote radioresistance by preferential activation of the DNA damage response. Nature. 2006; 444:756-760. [PubMed: 17051156]

6. Terpstra W, et al. Fluorouracil selectively spares acute myeloid leukemia cells with long-term growth abilities in immunodeficient mice and in culture. Blood. 1996; 88:1944-1950. [PubMed: 8822911]

7. Wicha MS, et al. Cancer stem cells: an old idea--a paradigm shift. Cancer Res. 2006; 66:1883-1890. discussion 1895-1886. [PubMed: 16488983]

8. Li Z, et al. Hypoxia-inducible factors regulate tumorigenic capacity of glioma stem cells. Cancer Cell. 2009; 15:501-513. [PubMed: 19477429]

9. Wang Y, et al. Targeting HIF1alpha Eliminates Cancer Stem Cells in Hematological Malignancies. Cell Stem Cell. 2011; 8:399-411. [PubMed: 21474104] 
10. Wang YH, et al. Cell-state-specific metabolic dependency in hematopoiesis and leukemogenesis. Cell. 2014; 158:1309-1323. [PubMed: 25215489]

11. Palazon A, et al. HIF transcription factors, inflammation, and immunity. Immunity. 2014; 41:518528. [PubMed: 25367569]

12. Cheng SC, et al. mTOR- and HIF-1alpha-mediated aerobic glycolysis as metabolic basis for trained immunity. Science. 2014; 345:1250684. [PubMed: 25258083]

13. Saeed S, et al. Epigenetic programming of monocyte-to-macrophage differentiation and trained innate immunity. Science. 2014; 345:1251086. [PubMed: 25258085]

14. Keith B, et al. HIF1alpha and HIF2alpha: sibling rivalry in hypoxic tumour growth and progression. Nat Rev Cancer. 2012; 12:9-22. [PubMed: 22169972]

15. Semenza GL. Hypoxia-inducible factors in physiology and medicine. Cell. 2012; 148:399-408. [PubMed: 22304911]

16. Wang GL, et al. Hypoxia-inducible factor 1 is a basic-helix-loop-helix-PAS heterodimer regulated by cellular O2 tension. Proc. Natl. Acad. Sci. U. S. A. 1995; 92:5510-5514. [PubMed: 7539918]

17. Luo W, et al. Pyruvate kinase M2 is a PHD3-stimulated coactivator for hypoxia-inducible factor 1. Cell. 2011; 145:732-744. [PubMed: 21620138]

18. Zhang P, et al. Hypoxia-inducible factor 3 is an oxygen-dependent transcription activator and regulates a distinct transcriptional response to hypoxia. Cell reports. 2014; 6:1110-1121. [PubMed: 24613356]

19. Kondo K, et al. Inhibition of HIF is necessary for tumor suppression by the von Hippel-Lindau protein. Cancer Cell. 2002; 1:237-246. [PubMed: 12086860]

20. Latif F, et al. Identification of the von Hippel-Lindau disease tumor suppressor gene. Science. 1993; 260:1317-1320. [PubMed: 8493574]

21. Maranchie JK, et al. The contribution of VHL substrate binding and HIF1-alpha to the phenotype of VHL loss in renal cell carcinoma. Cancer Cell. 2002; 1:247-255. [PubMed: 12086861]

22. Pereira T, et al. Identification of residues critical for regulation of protein stability and the transactivation function of the hypoxia-inducible factor-1alpha by the von Hippel-Lindau tumor suppressor gene product. J. Biol. Chem. 2003; 278:6816-6823. [PubMed: 12468553]

23. Zbar B. Von Hippel-Lindau disease and sporadic renal cell carcinoma. Cancer Surv. 1995; 25:219232. [PubMed: 8718521]

24. Semenza GL. HIF-1: upstream and downstream of cancer metabolism. Curr. Opin. Genet. Dev. 2010; 20:51-56. [PubMed: 19942427]

25. $\mathrm{Xu} \mathrm{W}$, et al. Oncometabolite 2-hydroxyglutarate is a competitive inhibitor of alpha-ketoglutaratedependent dioxygenases. Cancer Cell. 2011; 19:17-30. [PubMed: 21251613]

26. Zhao S, et al. Glioma-derived mutations in IDH1 dominantly inhibit IDH1 catalytic activity and induce HIF-1alpha. Science. 2009; 324:261-265. [PubMed: 19359588]

27. Ward PS, et al. The common feature of leukemia-associated IDH1 and IDH2 mutations is a neomorphic enzyme activity converting alpha-ketoglutarate to 2-hydroxyglutarate. Cancer Cell. 2010; 17:225-234. [PubMed: 20171147]

28. Pardanani A, et al. IDH1 and IDH2 mutation analysis in chronic- and blast-phase myeloproliferative neoplasms. Leukemia. 2010; 24:1146-1151. [PubMed: 20410924]

29. Abbas S, et al. Acquired mutations in the genes encoding IDH1 and IDH2 both are recurrent aberrations in acute myeloid leukemia: prevalence and prognostic value. Blood. 2010; 116:21222126. [PubMed: 20538800]

30. Paschka P, et al. IDH1 and IDH2 mutations are frequent genetic alterations in acute myeloid leukemia and confer adverse prognosis in cytogenetically normal acute myeloid leukemia with NPM1 mutation without FLT3 internal tandem duplication. J. Clin. Oncol. 2010; 28:3636-3643. [PubMed: 20567020]

31. Yan H, et al. IDH1 and IDH2 mutations in gliomas. N. Engl. J. Med. 2009; 360:765-773. [PubMed: 19228619]

32. Koivunen $\mathrm{P}$, et al. Transformation by the (R)-enantiomer of 2-hydroxyglutarate linked to EGLN activation. Nature. 2012; 483:484-488. [PubMed: 22343896]

Trends Pharmacol Sci. Author manuscript; available in PMC 2016 June 01. 
33. Bertout JA, et al. Heterozygosity for hypoxia inducible factor 1alpha decreases the incidence of thymic lymphomas in a p53 mutant mouse model. Cancer Res. 2009; 69:3213-3220. [PubMed: 19293180]

34. Lidgren A, et al. The expression of hypoxia-inducible factor 1alpha is a favorable independent prognostic factor in renal cell carcinoma. Clin. Cancer Res. 2005; 11:1129-1135. [PubMed: 15709180]

35. Klatte T, et al. Hypoxia-inducible factor 1 alpha in clear cell renal cell carcinoma. Clin. Cancer Res. 2007; 13:7388-7393. [PubMed: 18094421]

36. Mazumdar J, et al. HIF-2alpha deletion promotes Kras-driven lung tumor development. Proc. Natl. Acad. Sci. U. S. A. 2010; 107:14182-14187. [PubMed: 20660313]

37. Velasco-Hernandez T, et al. HIF-1alpha can act as a tumor suppressor gene in murine acute myeloid leukemia. Blood. 2014; 124:3597-3607. [PubMed: 25267197]

38. Zhang H, et al. HIF-1 inhibits mitochondrial biogenesis and cellular respiration in VHL-deficient renal cell carcinoma by repression of C-MYC activity. Cancer Cell. 2007; 11:407-420. [PubMed: 17482131]

39. Mazure NM, et al. Oncogenic transformation and hypoxia synergistically act to modulate vascular endothelial growth factor expression. Cancer Res. 1996; 56:3436-3440. [PubMed: 8758908]

40. Arany Z, et al. An essential role for $\mathrm{p} 300 / \mathrm{CBP}$ in the cellular response to hypoxia. Proc. Natl. Acad. Sci. U. S. A. 1996; 93:12969-12973. [PubMed: 8917528]

41. Forsythe JA, et al. Activation of vascular endothelial growth factor gene transcription by hypoxiainducible factor 1. Mol. Cell. Biol. 1996; 16:4604-4613. [PubMed: 8756616]

42. Kong D, et al. Echinomycin, a small-molecule inhibitor of hypoxia-inducible factor-1 DNAbinding activity. Cancer Res. 2005; 65:9047-9055. [PubMed: 16204079]

43. Comerford KM, et al. Hypoxia-inducible factor-1-dependent regulation of the multidrug resistance (MDR1) gene. Cancer Res. 2002; 62:3387-3394. [PubMed: 12067980]

44. Ricker JL, et al. 2-methoxyestradiol inhibits hypoxia-inducible factor 1alpha, tumor growth, and angiogenesis and augments paclitaxel efficacy in head and neck squamous cell carcinoma. Clin. Cancer Res. 2004; 10:8665-8673. [PubMed: 15623651]

45. Zhang H, et al. Kruppel-like factor 8 contributes to hypoxia-induced MDR in gastric cancer cells. Cancer science. 2014; 105:1109-1115. [PubMed: 25040744]

46. Ria R, et al. HIF-1alpha of bone marrow endothelial cells implies relapse and drug resistance in patients with multiple myeloma and may act as a therapeutic target. Clin. Cancer Res. 2014; 20:847-858. [PubMed: 24297864]

47. Li DW, et al. Hypoxia induced multidrug resistance of laryngeal cancer cells via hypoxia-inducible factor-1alpha. Asian Pacific journal of cancer prevention : APJCP. 2013; 14:4853-4858. [PubMed: 24083758]

48. Wang H, et al. Wogonin reverses hypoxia resistance of human colon cancer HCT116 cells via downregulation of HIF-1alpha and glycolysis, by inhibiting PI3K/Akt signaling pathway. Mol. Carcinog. 2014; 53(Suppl 1):E107-E118. [PubMed: 23761018]

49. Cui XY, et al. Hypoxia influences stem cell-like properties in multidrug resistant K562 leukemic cells. Blood Cells. Mol. Dis. 2013; 51:177-184. [PubMed: 23725749]

50. Ji Z, et al. Expression of MDR1, HIF-1alpha and MRP1 in sacral chordoma and chordoma cell line CM-319. J. Exp. Clin. Cancer Res. 2010; 29:158. [PubMed: 21143841]

51. Ding Z, et al. Expression and significance of hypoxia-inducible factor-1 alpha and MDR1/Pglycoprotein in human colon carcinoma tissue and cells. J. Cancer Res. Clin. Oncol. 2010; 136:1697-1707. [PubMed: 20217131]

52. An WG, et al. Stabilization of wild-type p53 by hypoxia-inducible factor 1alpha. Nature. 1998; 392:405-408. [PubMed: 9537326]

53. Shoshani T, et al. Identification of a novel hypoxia-inducible factor 1-responsive gene, RTP801, involved in apoptosis. Mol. Cell. Biol. 2002; 22:2283-2293. [PubMed: 11884613]

54. Bruick RK. Expression of the gene encoding the proapoptotic Nip3 protein is induced by hypoxia. Proc. Natl. Acad. Sci. U. S. A. 2000; 97:9082-9087. [PubMed: 10922063] 
55. Sowter HM, et al. HIF-1-dependent regulation of hypoxic induction of the cell death factors BNIP3 and NIX in human tumors. Cancer Res. 2001; 61:6669-6673. [PubMed: 11559532]

56. Koshiji M, et al. HIF-1alpha induces cell cycle arrest by functionally counteracting Myc. EMBO J. 2004; 23:1949-1956. [PubMed: 15071503]

57. Jin L, et al. Targeting of CD44 eradicates human acute myeloid leukemic stem cells. Nat. Med. 2006; 12:1167-1174. [PubMed: 16998484]

58. Jin L, et al. Monoclonal antibody-mediated targeting of CD123, IL-3 receptor alpha chain, eliminates human acute myeloid leukemic stem cells. Cell Stem Cell. 2009; 5:31-42. [PubMed: 19570512]

59. Kikushige Y, et al. TIM-3 is a promising target to selectively kill acute myeloid leukemia stem cells. Cell Stem Cell. 2010; 7:708-717. [PubMed: 21112565]

60. Guzman ML, et al. An orally bioavailable parthenolide analog selectively eradicates acute myelogenous leukemia stem and progenitor cells. Blood. 2007; 110:4427-4435. [PubMed: 17804695]

61. Kelly PN, et al. Tumor growth need not be driven by rare cancer stem cells. Science. 2007; 317:337. [PubMed: 17641192]

62. Wang L, et al. FISH+CD34+CD38- cells detected in newly diagnosed acute myeloid leukemia patients can predict the clinical outcome. Journal of hematology \& oncology. 2013; 6:85. [PubMed: 24517186]

63. Witte KE, et al. High proportion of leukemic stem cells at diagnosis is correlated with unfavorable prognosis in childhood acute myeloid leukemia. Pediatr. Hematol. Oncol. 2011; 28:91-99. [PubMed: 21214408]

64. van Rhenen A, et al. High stem cell frequency in acute myeloid leukemia at diagnosis predicts high minimal residual disease and poor survival. Clin. Cancer Res. 2005; 11:6520-6527. [PubMed: 16166428]

65. Gentles AJ, et al. Association of a leukemic stem cell gene expression signature with clinical outcomes in acute myeloid leukemia. JAMA. 2010; 304:2706-2715. [PubMed: 21177505]

66. NIH Clinical Center open to collaboration. Cancer discovery. 2014; 4:752.

67. Wang Y, et al. Echinomycin protects mice against relapsed acute myeloid leukemia without adverse effect on hematopoietic stem cells. Blood. 2014; 124:1127-1135. [PubMed: 24994068]

68. Bernot KM, et al. Toward personalized therapy in AML: in vivo benefit of targeting aberrant epigenetics in MLL-PTD-associated AML. Leukemia. 2013; 27:2379-2382. [PubMed: 23660685]

69. Sloma I, et al. Insights into the stem cells of chronic myeloid leukemia. Leukemia. 2010; 24:18231833. [PubMed: 20861912]

70. Mayerhofer M, et al. BCR/ABL induces expression of vascular endothelial growth factor and its transcriptional activator, hypoxia inducible factor-1alpha, through a pathway involving phosphoinositide 3-kinase and the mammalian target of rapamycin. Blood. 2002; 100:3767-3775. [PubMed: 12393646]

71. Zhao F, et al. Imatinib resistance associated with BCR-ABL upregulation is dependent on HIF-1alpha-induced metabolic reprograming. Oncogene. 2010; 29:2962-2972. [PubMed: 20228846]

72. Zhang H, et al. HIF1alpha is required for survival maintenance of chronic myeloid leukemia stem cells. Blood. 2012; 119:2595-2607. [PubMed: 22275380]

73. Ng KP, et al. Physiologic hypoxia promotes maintenance of CML stem cells despite effective BCR-ABL1 inhibition. Blood. 2014; 123:3316-3326. [PubMed: 24705490]

74. Conley SJ, et al. Antiangiogenic agents increase breast cancer stem cells via the generation of tumor hypoxia. Proc. Natl. Acad. Sci. U. S. A. 2012; 109:2784-2789. [PubMed: 22308314]

75. Schwab LP, et al. Hypoxia-inducible factor 1alpha promotes primary tumor growth and tumorinitiating cell activity in breast cancer. Breast cancer research : BCR. 2012; 14:R6. [PubMed: 22225988]

76. Cordenonsi M, et al. The Hippo transducer TAZ confers cancer stem cell-related traits on breast cancer cells. Cell. 2011; 147:759-772. [PubMed: 22078877] 
77. Xiang L, et al. Hypoxia-inducible factor 1 mediates TAZ expression and nuclear localization to induce the breast cancer stem cell phenotype. Oncotarget. 2014; 5:12509-12527. [PubMed: 25587023]

78. Chaturvedi $\mathrm{P}$, et al. Hypoxia-inducible factor-dependent signaling between triple-negative breast cancer cells and mesenchymal stem cells promotes macrophage recruitment. Proc. Natl. Acad. Sci. U. S. A. 2014; 111:E2120-E2129. [PubMed: 24799675]

79. Lukashev D, et al. Differential regulation of two alternatively spliced isoforms of hypoxiainducible factor-1 alpha in activated T lymphocytes. J. Biol. Chem. 2001; 276:48754-48763. [PubMed: 11602577]

80. Lukashev D, et al. Cutting edge: hypoxia-inducible factor 1alpha and its activation-inducible short isoform I.1 negatively regulate functions of CD4+ and CD8+ T lymphocytes. J. Immunol. 2006; 177:4962-4965. [PubMed: 17015677]

81. Dang EV, et al. Control of T(H)17/T(reg) balance by hypoxia-inducible factor 1. Cell. 2011; 146:772-784. [PubMed: 21871655]

82. Shi LZ, et al. HIF1alpha-dependent glycolytic pathway orchestrates a metabolic checkpoint for the differentiation of TH17 and Treg cells. J. Exp. Med. 2011; 208:1367-1376. [PubMed: 21708926]

83. Kryczek I, et al. Human TH17 cells are long-lived effector memory cells. Sci Transl Med. 2011; 3:104ra100.

84. Ben-Shoshan J, et al. Hypoxia controls CD4+CD25+ regulatory T-cell homeostasis via hypoxiainducible factor-1alpha. Eur. J. Immunol. 2008; 38:2412-2418. [PubMed: 18792019]

85. Facciabene A, et al. Tumour hypoxia promotes tolerance and angiogenesis via CCL28 and T(reg) cells. Nature. 2011; 475:226-230. [PubMed: 21753853]

86. Finlay DK, et al. PDK1 regulation of mTOR and hypoxia-inducible factor 1 integrate metabolism and migration of CD8+ T cells. J. Exp. Med. 2012; 209:2441-2453. [PubMed: 23183047]

87. Doedens AL, et al. Hypoxia-inducible factors enhance the effector responses of CD8(+) T cells to persistent antigen. Nat Immunol. 2013; 14:1173-1182. [PubMed: 24076634]

88. Thiel M, et al. Targeted deletion of HIF-1alpha gene in T cells prevents their inhibition in hypoxic inflamed tissues and improves septic mice survival. PLoS One. 2007; 2:e853. [PubMed: 17786224]

89. Jantsch J, et al. Hypoxia and hypoxia-inducible factor-1 alpha modulate lipopolysaccharideinduced dendritic cell activation and function. J. Immunol. 2008; 180:4697-4705. [PubMed: 18354193]

90. Noman MZ, et al. PD-L1 is a novel direct target of HIF-1alpha, and its blockade under hypoxia enhanced MDSC-mediated T cell activation. J. Exp. Med. 2014; 211:781-790. [PubMed: 24778419]

91. Barsoum IB, et al. A mechanism of hypoxia-mediated escape from adaptive immunity in cancer cells. Cancer Res. 2014; 74:665-674. [PubMed: 24336068]

92. Curiel TJ, et al. Blockade of B7-H1 improves myeloid dendritic cell-mediated antitumor immunity. Nat. Med. 2003; 9:562-567. [PubMed: 12704383]

93. Gabrilovich DI, Nagaraj S. Myeloid-derived suppressor cells as regulators of the immune system. Nat Rev Immunol. 2009; 9:162-174. [PubMed: 19197294]

94. Talmadge JE, Gabrilovich DI. History of myeloid-derived suppressor cells. Nat Rev Cancer. 2013; 13:739-752. [PubMed: 24060865]

95. Corzo CA, et al. HIF-1alpha regulates function and differentiation of myeloid-derived suppressor cells in the tumor microenvironment. J. Exp. Med. 2010; 207:2439-2453. [PubMed: 20876310]

96. Doedens AL, et al. Macrophage expression of hypoxia-inducible factor-1 alpha suppresses T-cell function and promotes tumor progression. Cancer Res. 2010; 70:7465-7475. [PubMed: 20841473]

97. Chen W, et al. mTOR signaling is activated by FLT3 kinase and promotes survival of FLT3mutated acute myeloid leukemia cells. Mol Cancer. 2010; 9:292. [PubMed: 21067588]

98. Gilliland DG, Griffin JD. The roles of FLT3 in hematopoiesis and leukemia. Blood. 2002; 100:1532-1542. [PubMed: 12176867]

99. Sathaliyawala T, et al. Mammalian target of rapamycin controls dendritic cell development downstream of Flt3 ligand signaling. Immunity. 2010; 33:597-606. [PubMed: 20933441] 
100. Brugarolas J, et al. Regulation of mTOR function in response to hypoxia by REDD1 and the TSC1/TSC2 tumor suppressor complex. Genes Dev. 2004; 18:2893-2904. [PubMed: 15545625]

101. Brugarolas JB, et al. TSC2 regulates VEGF through mTOR-dependent and -independent pathways. Cancer Cell. 2003; 4:147-158. [PubMed: 12957289]

102. Hudson CC, et al. Regulation of hypoxia-inducible factor 1alpha expression and function by the mammalian target of rapamycin. Mol. Cell. Biol. 2002; 22:7004-7014. [PubMed: 12242281]

103. Van de Velde S, et al. mTOR links incretin signaling to HIF induction in pancreatic beta cells. Proc. Natl. Acad. Sci. U. S. A. 2011; 108:16876-16882. [PubMed: 21949366]

104. Huang J, et al. Pivotal role for glycogen synthase kinase-3 in hematopoietic stem cell homeostasis in mice. J. Clin. Invest. 2009; 119:3519-3529. [PubMed: 19959876]

105. Parkin B, et al. Acquired genomic copy number aberrations and survival in adult acute myelogenous leukemia. Blood. 2010; 116:4958-4967. [PubMed: 20729466]

106. Joshi S, et al. MDM2 regulates hypoxic hypoxia-inducible factor 1alpha stability in an E3 ligase, proteasome, and PTEN-phosphatidylinositol 3-kinase-AKT-dependent manner. J. Biol. Chem. 2014; 289:22785-22797. [PubMed: 24982421]

107. Ravi R, et al. Regulation of tumor angiogenesis by p53-induced degradation of hypoxia-inducible factor 1alpha. Genes Dev. 2000; 14:34-44. [PubMed: 10640274]

108. Falini B, et al. Cytoplasmic nucleophosmin in acute myelogenous leukemia with a normal karyotype. N. Engl. J. Med. 2005; 352:254-266. [PubMed: 15659725]

109. Grisendi S, Pandolfi PP. NPM mutations in acute myelogenous leukemia. N. Engl. J. Med. 2005; 352:291-292. [PubMed: 15659732]

110. Chen D, et al. Transcription-independent ARF regulation in oncogenic stress-mediated p53 responses. Nature. 2010; 464:624-627. [PubMed: 20208519]

111. Fatyol K, Szalay AA. The p14ARF tumor suppressor protein facilitates nucleolar sequestration of hypoxia-inducible factor-1alpha (HIF-1alpha) and inhibits HIF-1-mediated transcription. J. Biol. Chem. 2001; 276:28421-28429. [PubMed: 11382768]

112. Wang H, et al. Negative regulation of Hif1a expression and TH17 differentiation by the hypoxiaregulated microRNA miR-210. Nat Immunol. 2014; 15:393-401. [PubMed: 24608041]

113. Blouin CC, et al. Hypoxic gene activation by lipopolysaccharide in macrophages: implication of hypoxia-inducible factor 1alpha. Blood. 2004; 103:1124-1130. [PubMed: 14525767]

114. Laughner E, et al. HER2 (neu) signaling increases the rate of hypoxia-inducible factor 1alpha (HIF-1alpha) synthesis: novel mechanism for HIF-1-mediated vascular endothelial growth factor expression. Mol. Cell. Biol. 2001; 21:3995-4004. [PubMed: 11359907]

115. Cui TX, et al. Myeloid-derived suppressor cells enhance stemness of cancer cells by inducing microRNA101 and suppressing the corepressor CtBP2. Immunity. 2013; 39:611-621. [PubMed: 24012420]

116. Kryczek I, et al. IL-22(+)CD4(+) T cells promote colorectal cancer stemness via STAT3 transcription factor activation and induction of the methyltransferase DOT1L. Immunity. 2014; 40:772-784. [PubMed: 24816405]

117. Lin EY, et al. Macrophages regulate the angiogenic switch in a mouse model of breast cancer. Cancer Res. 2006; 66:11238-11246. [PubMed: 17114237]

118. Ben-Neriah Y, Karin M. Inflammation meets cancer, with NF-kappaB as the matchmaker. Nat Immunol. 2011; 12:715-723. [PubMed: 21772280]

119. Lin EY, Pollard JW. Tumor-associated macrophages press the angiogenic switch in breast cancer. Cancer Res. 2007; 67:5064-5066. [PubMed: 17545580] 


\section{Highlights}

Hypoxia-inducible factors maintain cancer stem cells

Hypoxia-inducible factors set the cancer microenvironment

Genetic and epigenetic mechanisms confers oxygen-resistance of hypoxia-inducible factors

Hypoxia-inducible factors are targets for therapeutic elimination of cancer stem cells and reprograming of the tumor microenvironment. 


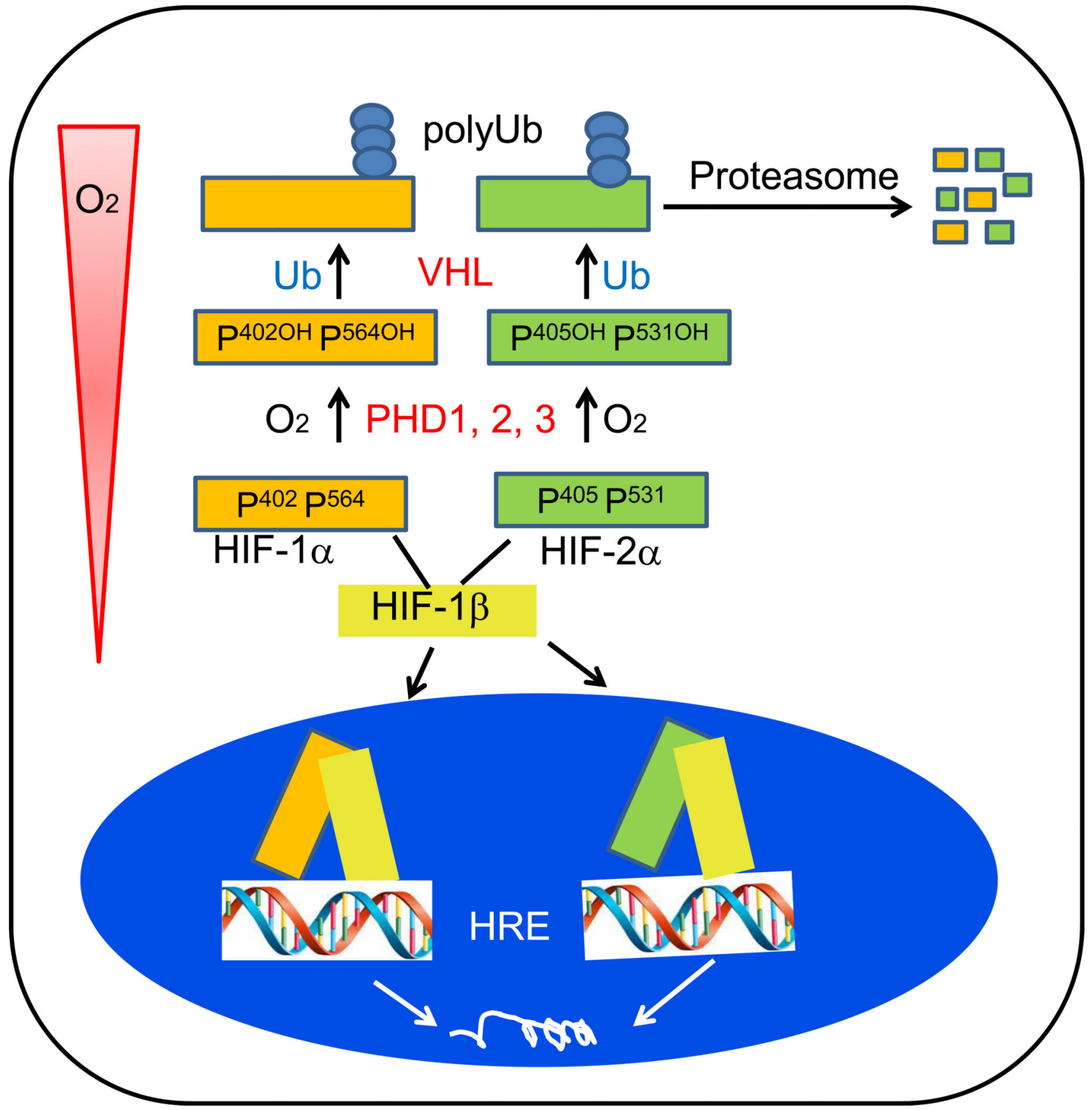

Fig. 1.

Regulation of hypoxia-inducible factors (HIF) activity in response to oxygen levels. Under hypoxic conditions, stablilized HIF-1a and HIF-2 $\alpha$ dimerize with HIF-1 $\beta$. The heterodimers translocate into the nucleus to regulate gene transcription. In the presence of oxygen, HIF-1a and HIF-2a proteins are hydroxylated by the prolyl hydroxylase domain protein (PHD)1-3 at the indicated proline residues. Hydroxylated HIF is recognized by VHL, which causes polyubiquitinylation and proteasome-mediated degradation of HIF. 


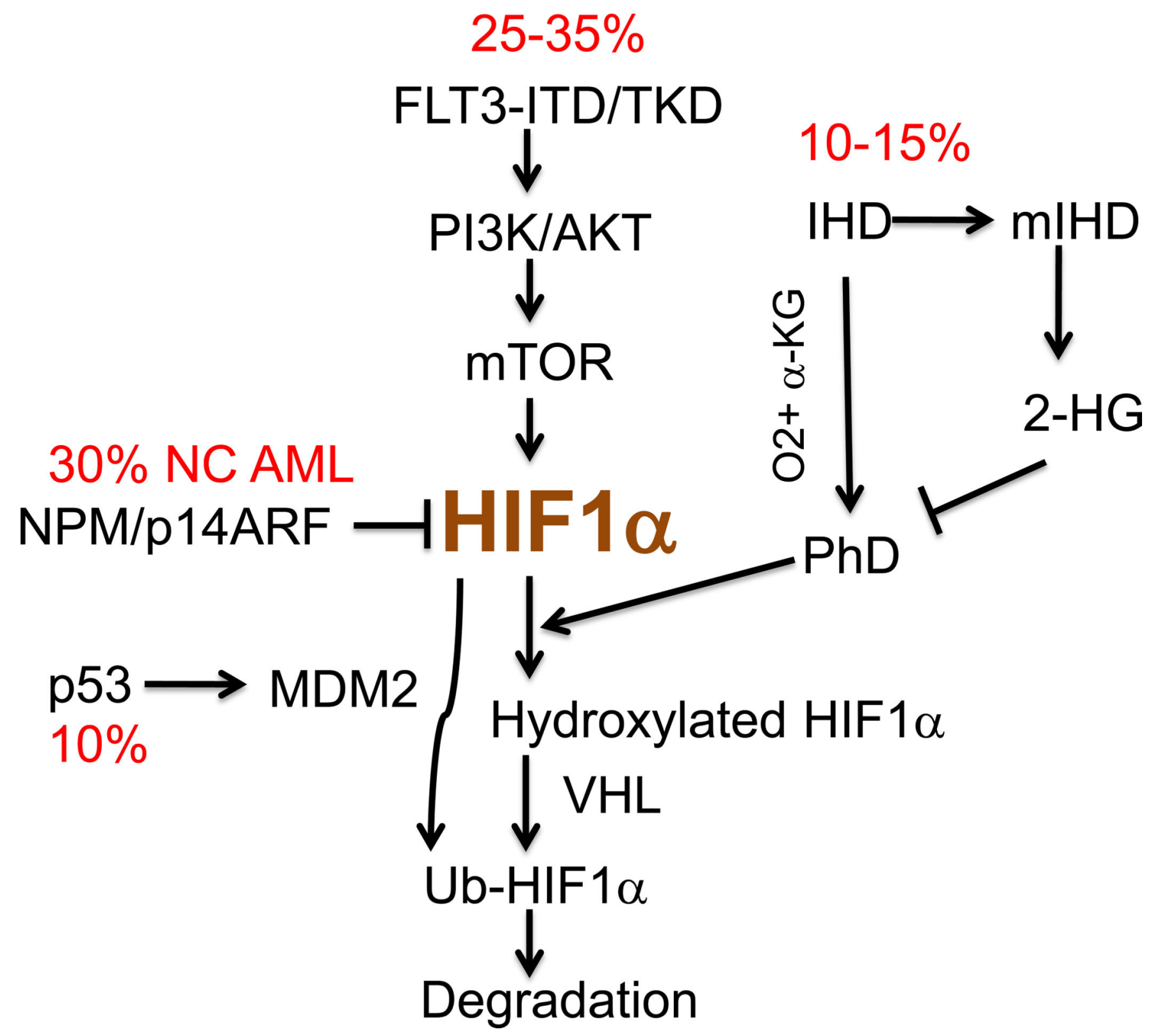

Fig. 2.

Prevalent mutations in acute myeloid leukemia (AML) potentially affect HIF levels in cancer cells. Mutations of genes encoding isocitrate dehydrogenase (IDH) 1 and 2, which are mutated in 10-15\% of AML, inactivates PHD to suppress HIF hydroxylation. FLT3 mutations (found in 25-35\% AML cases) stimulates PI3K/AKT pathway to enhance HIF translation. NPM mutation, which was found in 30\% of AML with normal karyotype (NC), reduces p14ARF, and HIF inhibitors. TP53 mutation reduces expression of MDM2, an alternative E3 ubiquitinylation ligase E3 for HIF. This in turn may reduce HIF ubiquitinylation. 


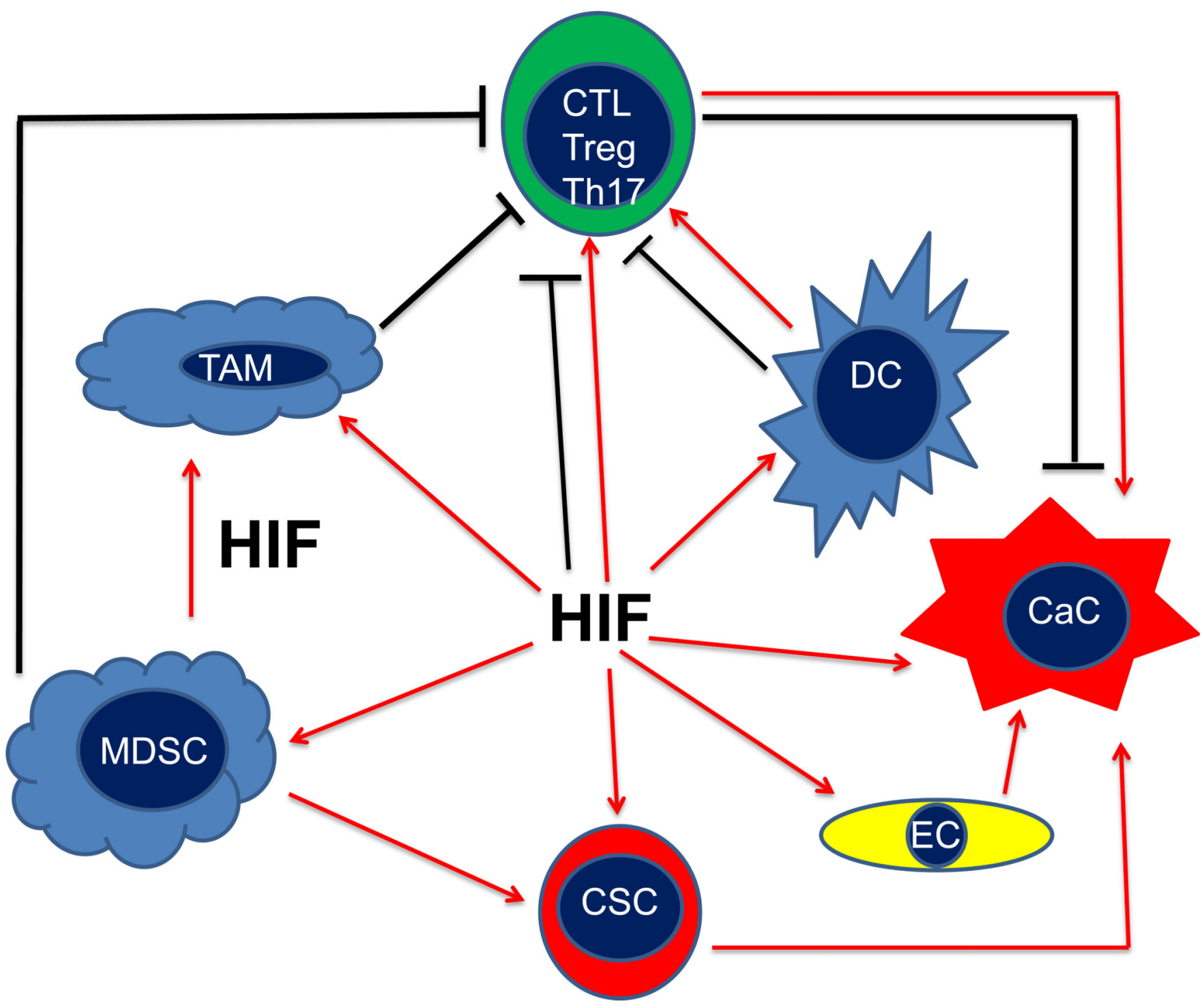

Fig. 3.

A central role for HIF in tumor microenvironment. In addition to its critical role for maintenance and proliferation of cancer stem cells (CSC) and cancer cells (CaC), HIF promotes differentiation and/or proliferation of host cells within the tumor microenvironment, including endothelial cells (EC), dendritic cells (DC), tumor-associated macrophages (TAM) and myeloid-derived suppressor cells (MDSC). Apart from regulating cells that affect $\mathrm{T}$ cell function, HIF also directly regulates activation and differentiation of various functional subsets of $\mathrm{T}$ cells, including cytolytic $\mathrm{T}$ lymophocytes (CTL), regulatory $\mathrm{T}$ cells (Treg), and IL-17-producing T cells (Th17). 